\title{
Mus musculus-microRNA-449a ameliorates neuropathic pain by decreasing the level of KCNMA1 and TRPA1, and increasing the level of TPTE
}

\author{
SHAN LU ${ }^{1}$, SICHAO MA $^{2}$, YUNYUN WANG $^{1}$, TAO HUANG $^{3}$, ZHIHUA ZHU $^{1}$ and GUOQING ZHAO ${ }^{1}$ \\ ${ }^{1}$ Department of Anesthesia, China-Japan Union Hospital of Jilin University, Changchun, Jilin 130033; Departments of \\ ${ }^{2}$ Nephrology and ${ }^{3}$ Emergency, The First Affiliated Hospital to Changchun University of Chinese Medicine, \\ Changchun, Jilin 130021, P.R. China
}

Received February 25, 2016; Accepted March 13, 2017

DOI: $10.3892 / \mathrm{mmr} .2017 .6559$

\begin{abstract}
Neuropathic pain is a nerve disorder characterized by the dysregulation of ion channels in dorsal root ganglion (DRG) neurons. MicroRNAs (miRs) may be associated with the molecular mechanisms underlying the altered levels of ion channels; however, the molecular mechanisms remain widely unknown. To investigate these mechanisms, the present study conducted a genomic analysis of miR between a unilateral spared nerve injury (SNI) model and sham control. Differentially expressed miRs between the SNI and sham groups were selected for transfection of DRG cells, a polymerase chain reaction (PCR) array analysis was subsequently performed. A total of three significantly differently expressed genes were selected from the results of the PCR array and further analyzed by reverse transcription-quantitative PCR. Genomic analysis revealed that Mus musculus miR-449a (mmu-miR-449a) was reduced in the SNI groups compared with the sham controls. The PCR array indicated that mmu-miR-449a-transfection reduced the mRNA expression levels of transient receptor potential cation channel subfamily A member 1 (TRPA1), and calcium-activated potassium channel subunit $\alpha-1$ (KCNMA1) and increased the level of transmembrane phosphatase with tension homology (TPTE) in the DRG cells $(\mathrm{P}<0.05)$. qRT-PCR analysis further indicated that mmu-miR-449a transfection caused similar alterations in the mRNA expression levels of TRPA1, KCNMA1 and TPTE in DRG cells, respectively $(\mathrm{P}<0.05)$. Therefore, mmu-miR-449a may ameliorate neuropathic pain by decreasing the activity of the channel proteins TRPA1 and KCNMA1 and increasing
\end{abstract}

Correspondence to: Dr Guoqing Zhao, Department of Anesthesia, China-Japan Union Hospital of Jilin University, 126 Xiantai Street, Changchun, Jilin 130033, P.R. China

E-mail: zhaoguoqing_ch@126.com

Key words: channel protein, type II $\beta$ subunit, Mus musculus miRNA-449a, dorsal root ganglia, neuropathic pain the levels of TPTE. mmu-miR-449a may be a potential therapeutic molecule for the alleviation of neuropathic pain.

\section{Introduction}

Pain is one of the most common health issues and produces substantial personal, community and global burdens (1-7). The majority of individuals have experienced or are experiencing pain. However, there remains no effective therapeutic agents for preventing pain. The majority of drugs used for treating pain have been known to have obvious side effects $(8,9)$. Therefore, it is necessary to examine the underlying mechanisms of pain and investigate novel therapeutic strategies for the treatment of pain.

Recent studies have demonstrated that inactivated ion channels and altered channel function results in hypersensitivity to painful stimuli (10-14). Peripheral neuropathic pain is a disorder caused by nerve injury, and is characterized by the dysregulation of voltage-gated ion channels, which are expressed in dorsal root ganglion (DRG) sensory neurons (15).

Previous studies have revealed that microRNAs (miRNAs/miRs) are involved in regulation of ion channels and affect sensitivity to painful stimuli $(16,17)$. miRNAs may be important small molecules associated with pain pathogenesis. miRNAs are a type of small, non-coding RNA, which act as post-transcriptional regulators of gene expression (18-21). Recent reports have identified that neural miRNAs serve critical roles at different stages of neuronal development, including neuritis outgrowth (22), dendritogenesis (23) and spine formation (24). Kusuda et al reported that miRNAs are involved in regulating the microRNA associated with the pathogenesis of chronic pain (25). Reverse transcription-quantitative polymerase chain reaction (RT-qPCR) analyses have demonstrated that miRNA (miR)-1, -16 and -206 are expressed differentially in DRG neurons and the dorsal horn of the spinal cord in different painful situations. Furthermore, complete Freud's adjuvant-induced inflammation significantly reduced miR-1,-16 and -206 levels in a time-dependent manner. By contrast, miRNAs were upregulated in the spinal dorsal horn (25). The association between miRNA expression levels and numerous pathophysiological processes implicates their role in neuropathic pain. 
With the development of genome libraries of miRNA mimics, it is possible to identify target miRNA using a high throughput screening method $(26,27)$. Therefore, the present study used this technique to investigate miRNAs associated with neuropathic pain, and investigated underlying molecular mechanisms.

\section{Materials and methods}

Animal model. All experimental procedures were approved by the Committee on Animal Experimentation of the China-Japan Union Hospital of Jilin University (Changchun, China). Adult male Kunming mice (weight, 18-22 g; $n=8$ ) were purchased from the Animal Center of Jilin University. Animals were housed in a $12 \mathrm{~h}$ light/dark cycle and had free access to food and water at $25^{\circ} \mathrm{C}$ and $50+/-10 \%$ relative humidity. Spared-nerve injury (SNI) was established according to a previous report, with slight modifications (28). SNI was established by the unilateral ligation and cutting of the peroneal and tibial nerve branches, while the sural nerve was intact on the left (SNI-L, $\mathrm{n}=2$ ) or right (SNI-R, $\mathrm{n}=2$ ) hind paws. Notably, only the lateral side of the paw was innervated by SNI; therefore, only this area developed neuropathy. In sham controls, all nerves and their branches were exposed but no branch was ligated or transected on the left (Sham-L, n=2) or right (Sham-R, n=2) hind paws. The wound was sutured and the mice were allowed to recover. The Von Frey test for mechanical allodynia was conducted as previously described by Richner et al (28) one day prior to surgery, and every two days following.

$D R G$ culture. A total of 8 male and 8 female Kunming mice (age, 8 weeks; weight, 18-22 g) were maintained by the China-Japan Union Hospital of Jilin University. Animals were housed in a $12 \mathrm{~h}$ light/dark cycle and had free access to food and water at $25^{\circ} \mathrm{C}$ and $50+/-10 \%$ relative humidity. Breeding and maintenance of the mouse colony were performed until embryos were formed. Embryos were sacrificed using ice-cold PBS followed by decapitation. DRGs from vertebral levels were isolated and immersed in ice-cold PBS. DRGs were digested in a solution of $0.6 \mathrm{mg} / \mathrm{ml}$ collagenase type IX (Sigma-Aldrich; Merck Millipore, Darmstadt, Germany) and $1 \mathrm{mg} / \mathrm{ml}$ dispase II (Sigma-Aldrich; Merck Millipore) at $37^{\circ} \mathrm{C}$ in $5 \% \mathrm{CO}_{2}$ and $95 \%$ air for $40 \mathrm{~min}$. Following titration, the DRGs were cultured in Dulbecco's modified Eagle's medium/nutrient mixture F-12 (Biofluids, Inc., Rockville, MD, USA) supplemented with $2 \mathrm{mM}$ glutamine and $10 \%$ fetal bovine serum (Hyclone; GE Healthcare Life Sciences, Logan, UT, USA). The medium was supplemented with $100 \mathrm{U} / \mathrm{ml}$ interferon gamma (R\&D Systems, Inc., Minneapolis, MN, USA) to promote the formation of cell lines. The cells were cultured at $37^{\circ} \mathrm{C}$ for 10 passages prior to cloning.

Establishment of DRG cell lines. Individual clones were isolated via cloning rings and diluted in 24 -well plates. The cell lines were routinely cultured in a proliferating condition and passaged every week. The clones were able to grow to $>50$ passages.

Total RNA isolation from DRGs. A total of 2 weeks following SNI surgery, DRGs were isolated according to a previous protocol (29). Each pool was added to $1 \mathrm{ml}$ TRIzol ${ }^{\circledR}$ reagent (Invitrogen; Thermo Fisher Scientific, Inc., Waltham, MA, USA) and homogenized with a homogenizer. Following isolation with chloroform, RNA was precipitated using isopropanol. The resultant pellets were re-suspended in Tris/EDTA buffer (10 mM Tris-HCl, 1 mM EDTA, pH 8.0). Following digestion with DNase, the quality and purity of RNA was measured using a Bioanalyzer system (Agilent Technologies, Inc., Santa Clara, CA, USA). RNA integrity and contaminated genomes were identified by agarose gel electrophoresis.

Microarray analysis. A TaqMan ${ }^{\circledR}$ Array Rodent MicroRNA Card was used in a 7900HT Fast Real-Time PCR system (both purchased from Applied Biosystems; Thermo Fisher Scientific, Inc.). The card consisted of 384 TaqMan MicroRNA assays, which enables the quantification of 226 miRs in mice. This work was conducted at KangChen Bio-tech Inc. (Shanghai, China). Mus musculus miRNA (mmu-miR)-449a and -185 DNA constructs demonstrated significant alterations between the SNI and sham hind paws $(\mathrm{P}<0.05$; Table I). Therefore, these two mmu-miRs were selected for the subsequent experiments. mmu-miR-449a (accession no. NR_029961) and mmu-miR-185 (accession no. NR_029706) were cloned into the NdeI-EcoRI sites of a pcDNA3.1 vector (Invitrogen; Thermo Fisher Scientific, Inc.) using a TOPO ${ }^{\circledR}$ Taq-Amplified Expression kit (Life Technologies; Thermo Fisher Scientific, Inc.), and subsequently named pcDNA3.1-miR-449a and pcDNA3.1-miR-185, respectively. Plasmids were amplified in E. coli topoisomerase-10, purified using a QIAprep ${ }^{\circledR}$ Miniprep kit (Qiagen, Inc., Valencia, CA, USA) and identified by sequencing.

Transfection of DRG cells. The DRG cell lines were transfected with $2 \mu \mathrm{g}$ pcDNA3.1-miR-449a or pcDNA3.1-miR-185 as treatment groups. Transfection was conducted in $>50 \%$ confluent cells in 6-well plates by Lipofectamine ${ }^{\circledR} 2000$ (Life Technologies; Thermo Fisher Scientific, Inc.).

Channel gene selection. Microarray analyses revealed that FXYD domain-containing ion transport regulator 3 (FXYD3), transient receptor potential cation channel subfamily A member 1 (TRPA1) and calcium-activated potassium channel subunit $\alpha-1$ (KCNMA1) mRNA expression levels were completely inhibited, whereas calcium channel, voltage-dependent L-type calcium channel subunit $\alpha-1 \mathrm{C}$ (CACNA1C), $\gamma$ subunit of the epithelial sodium channel and transmembrane phosphatase with tension homology (TPTE) mRNA expression levels were significantly increased in DRG cells transfected with mmu-miR-449a when compared with non-transfected cells. TRPA1 has been widely reported to be associated with pain pathogenesis $(14,30)$. KCNMA1 regulates the potassium channel (31), which has additionally been demonstrated to be associated with pain pathogenesis $(32,33)$. TPTE is a transmembrane phosphatase, which has tensin homology and reaches the highest level among all channel proteins. Therefore, the most differentially expressed mRNAs TRPA1, KCNMA1 and TPTE were selected for subsequent experiments.

$R T-q P C R$. A total of one month following SNI surgery, DRGs were isolated according to a previous protocol (29). RNA was 
Table I. Significantly dysregulated microRNAs in SNI and sham mice.

\begin{tabular}{lllcl}
\hline miRs & logFC & P-value & $\begin{array}{c}\text { Average Ct values } \\
\text { of miR levels (SNI) }\end{array}$ & $\begin{array}{c}\text { Average Ct values of } \\
\text { miR levels (Sham) }\end{array}$ \\
\hline mmu-miR-685 & 3.354 & 0.245957 & 8.268499971 & 6.167500019 \\
mmu-miR-337-3p & 1.7265 & 0.380265 & 9.698999882 & 8.222500086 \\
mmu-miR-139-3p & 1.4535 & 0.220282 & 4.266250015 & 3.332000137 \\
mmu-miR-687 & 1.357 & 0.370371 & 5.784999967 & 4.99000001 \\
mmu-miR-365 & -1.4085 & 0.225318 & 5.113250017 & 6.48149991 \\
mmu-miR-101a & -1.42 & 0.099638 & 6.218500018 & 7.390500069 \\
mmu-miR-339-5p & -1.5095 & 0.197606 & 5.355749965 & 6.210500002 \\
mmu-miR-130b & -2.002 & 0.144655 & 7.530249834 & 9.433000089 \\
mmu-miR-449a & -2.3225 & 0.039836 & 7.360249996 & 9.310999873 \\
mmu-miR-185 & -2.407 & 0.048612 & 6.308750033 & 8.404500151 \\
\hline
\end{tabular}

$\log \mathrm{FC}, \log$ of fold change; mmu, Mus musculus; miR, microRNA; SNI, spared-nerve injury. $\mathrm{P}<0.05$ was considered to indicate a statistically significant difference.

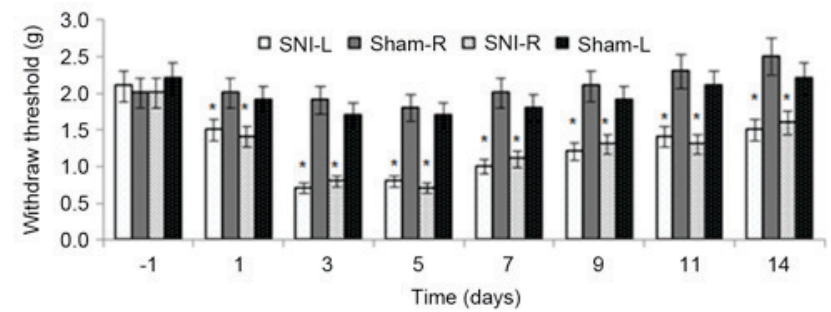

Figure 1. Von Frey test on Kunming mice prior to and following surgery. SNI was established by unilateral ligation and cutting of peroneal and the tibial nerve branches while the sural nerve was intact on the left (SNI-L) or right (SNI-R) hind paws. In sham controls, all the nerves and their branches were exposed but no branch was ligated or transected on the left (Sham-L) or right (Sham-R) hind paws. All data are presented as the mean \pm standard deviation ( $\mathrm{n}=4$ in each group). ${ }^{*} \mathrm{P}<0.05$ vs. the respective sham group. $\mathrm{SNI}$, spared-nerve injury; R, right L, left.

isolated using a Takara MiniBEST Universal RNA Extraction kit (code no. 9767; Takara Biotechnology Co., Ltd., Dalian, China). RNA (5 $\mu \mathrm{g})$ was reverse-transcribed using an RT kit (Takara Biotechnology Co., Ltd.). The mRNA expression levels of TPA1, KCNMA1 and TPTE were further measured in the mmu-miR-449a transfected and non-transfected DRG cell lines, SNI models and sham controls. Platinum ${ }^{\circledR}$ TaqDNA polymerase was purchased from Applied Biosystems; Thermo Fisher Scientific, Inc. qPCR with SYBR ${ }^{\circledR}$ Green (Invitrogen; Thermo Fisher Scientific, Inc.) detection was performed using Mouse Neuroscience Ion Channels and Transporters $\mathrm{RT}^{2}$ Profiler ${ }^{\mathrm{TM}}$ PCR Array containing 84 primer sets for ion channel and transporter genes (Qiagen, Inc.). qPCR was performed in triplicate using a SYBR Green PCR Master Mix with the following primers: Forward, 5'-gaactgatcatcaatggttc-3 and reverse, 5'-aggtttggatttgctccttg-3 for TRPA1; forward, 5'-aaa accttgagcgcaacag-3' and reverse, 5'-gcctaactctcaggtgctcc-3 for KCNMA1; forward, 5'-cctgctgaacgagggttcag-3' and reverse, 5'-tgtgcttggctctttccagc-3' for TPTE; forward, 5'-ttcccetccatc gtgggccg-3' and reverse, 5'-gtcccagttggtaacaatgc-3' for $\beta$-actin. Reactions were carried out using a 7500 Fast Real-Time PCR system (Applied Biosystems; Thermo Fisher Scientific, Inc.) with the following conditions: Initial denaturation at $95^{\circ} \mathrm{C}$ for $10 \mathrm{~min}$, followed by 40 cycles of denaturation at $95^{\circ} \mathrm{C}$ for $10 \mathrm{sec}$, annealing at $60^{\circ} \mathrm{C}$ for $34 \mathrm{sec}$ and extension at $60^{\circ} \mathrm{C}$ for $60 \mathrm{sec}$. The relative expression value was calculated by the $2^{\Delta \Delta \mathrm{Cq}}$ method (34).

Due to the small sample size, the experiment was repeated on 8 further Kunming male mice (age, 8 weeks; weight, 18-22 g). Mice were purchased from the Animal Center of Jilin University and maintained by the China-Japan Union Hospital of Jilin University, as aforementioned. SNI and sham surgeries were performed as already described.

Statistical analysis. To compare groups, one-way analysis of variance was performed, followed by Tukey's post hoc test. Statistical analyses were performed using GraphPad Prism software version 5.0.4 (GraphPad Software Inc., La Jolla, CA, USA). Data are expressed as the mean \pm standard deviation. ${ }^{*} \mathrm{P}<0.05$ was considered to indicate a statistically significant difference.

\section{Results}

Evaluation of the SNI mouse model. In the SNI mouse model, the onset of mechanical allodynia was assessed 1 day prior to and 2 days following surgery. Von Frey testing of SNI was performed on the SNI-L, SNI-R, Sham-R and Sham-L groups. The unilateral SNI hind paws maintained strong mechanical allodynia during the whole observation period of two weeks. The SNI hind paws developed obvious hypersensitivity on the operated paw $(\mathrm{P}<0.05)$, particularly at days 3 and 5 , whereas the sham hind paws were not significantly affected. The sham animals exhibited no significant alterations in the threshold for mechanical allodynia over time (Fig. 1). These results implied that a SNI model was successfully established.

Hierarchical cluster analysis of differentially dysregulated microRNAs in the SNI model and sham controls. The spectrum of the differentially dysregulated microRNAs was analyzed in the DRG cells of SNI models and sham animals 


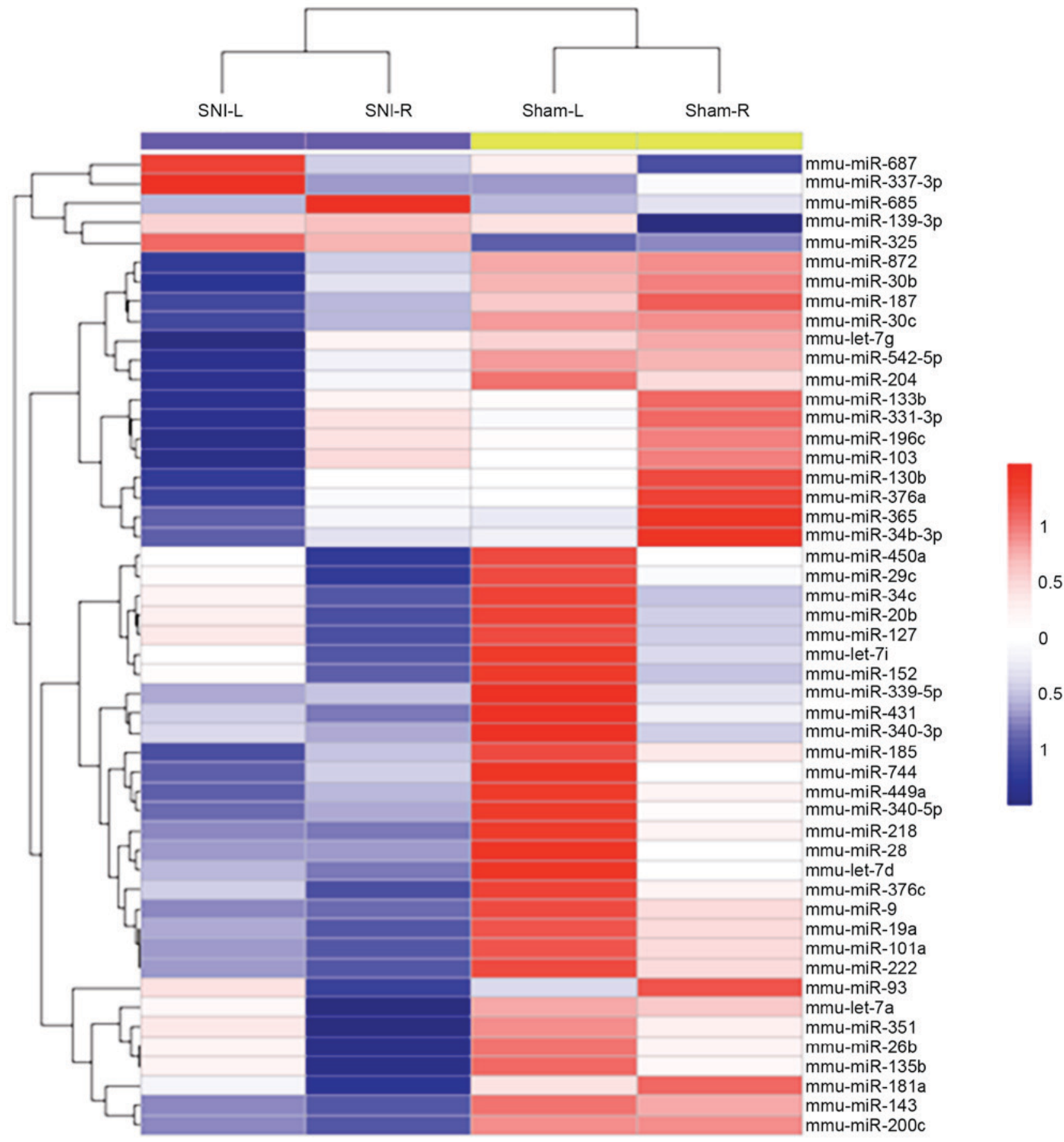

Figure 2. Hierarchical cluster analysis of differentially dysregulated microRNAs in SNI models and sham mice. The color code in each heat map is linear with blue as the lowest and red as the highest level. Upregulated microRNAs are presented as blue to red, and downregulated microRNAs are presented as red to blue. SNI, spared-nerve injury; R, right; L, left; mmu, Mus musculus; miR, microRNA.

within the context of the four mRNA-expression subtypes. Numerous differentially dysregulated microRNAs exhibited mRNA-subtype-specific and clinical-subtype-specific patterns. The differentially dysregulated microRNAs were considerably more diverse and recurrent within the SNI and sham groups. However, the overall upregulated microRNAs were increased in the sham groups and reduced in the SNI groups (Fig. 2).

Hierarchical cluster analysis of differentially dysregulated microRNAs revealed 154 dysregulated microRNAs in the sham groups and reduced levels in the SNI groups. The levels of mmu-miR-685, -337-3p, -139-3p, -687, -365, -101a, -339-5p,
$-130 b,-449 a$ and -185 were markedly increased in sham animals. Among these 10 microRNAs, only mmu-miR-449a and -185 had statistically significant alterations in SNI models compared with sham groups $(\mathrm{P}<0.05)$. No significant differences were observed in the expression levels of the additional 8 miRs assessed between the SNI and sham groups ( $\mathrm{P}>0.05$; Table I).

PCR array. PCR array analysis indicated that mmu-miR-449a transfection of DRG cells affected the mRNA expression levels of the majority of ion channel genes, compared with non-transfected cells (Fig. 3). For convenience, the 


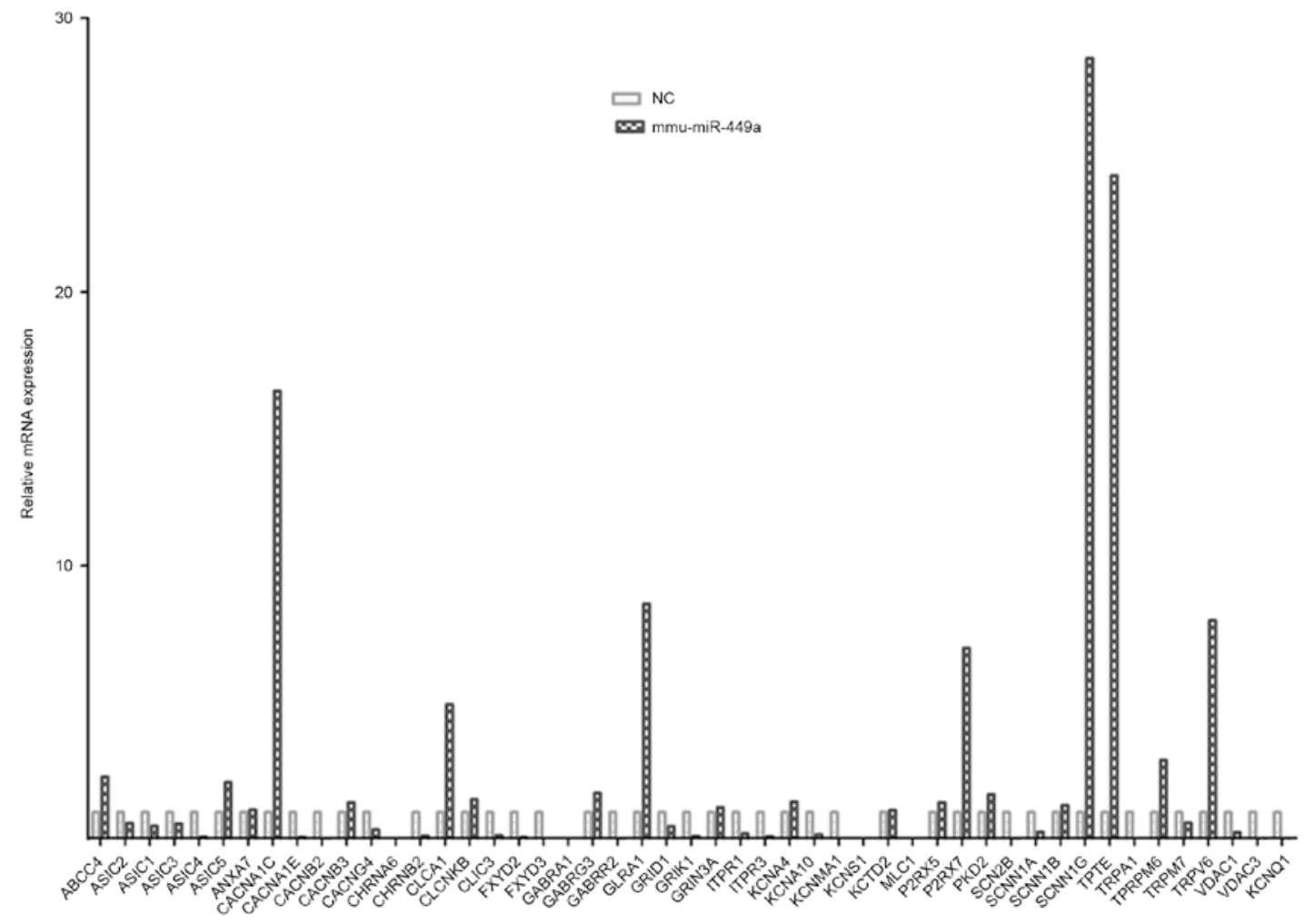

Figure 3. PCR array analysis of the effects of mmu-miR-449a on the mRNA expression levels of ion channels. PCR analysis of significantly altered ion channel encoding genes in dorsal root ganglion cells transfected with mmu-miR-449a. NC, non-transfected cells; mmu, Mus musculus; miR, microRNA; PCR, polymerase chain reaction.

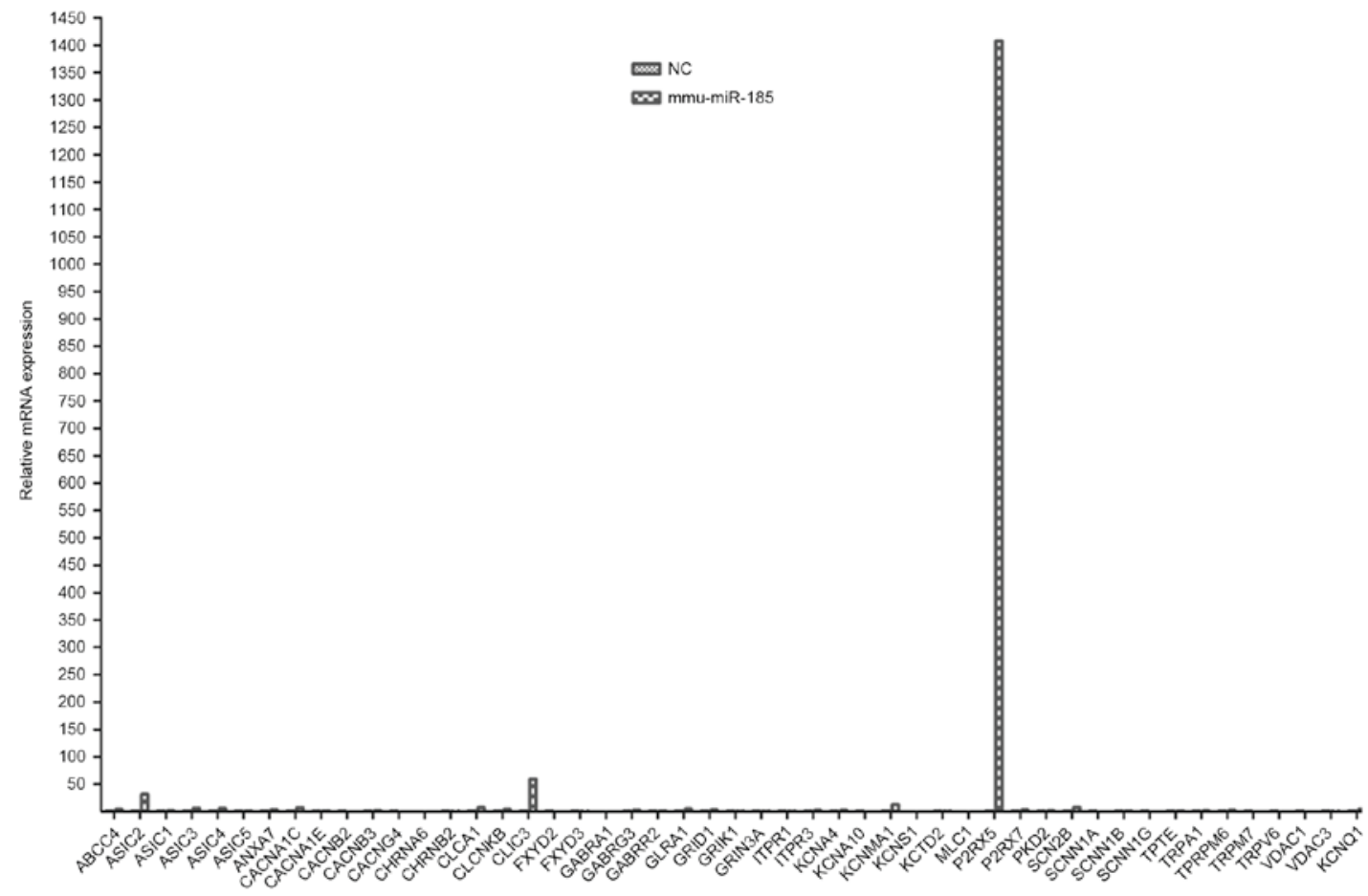

Figure 4. PCR array analysis of the effects of mmu-miR-185 on the levels of ion channels proteins. PCR array analysis of significantly altered ion channel encoding genes in dorsal root ganglion cell line cells transfected with mmu-miR-185. NC, non-transfected cells; mmu, Mus musculus; miR, microRNA; PCR, polymerase chain reaction.

three most differentially expressed ion channel genes were selected for subsequent experiments: TRPA1, KCNMA1 and TPTE $(\mathrm{P}<0.05)$. Unlike mmu-miR-449a, PCR array analysis demonstrated that mmu-miR-185 transfection did not alter the majority of ion channel encoding genes, except for the members of the P2X family of ligand-gated cation channels, including 


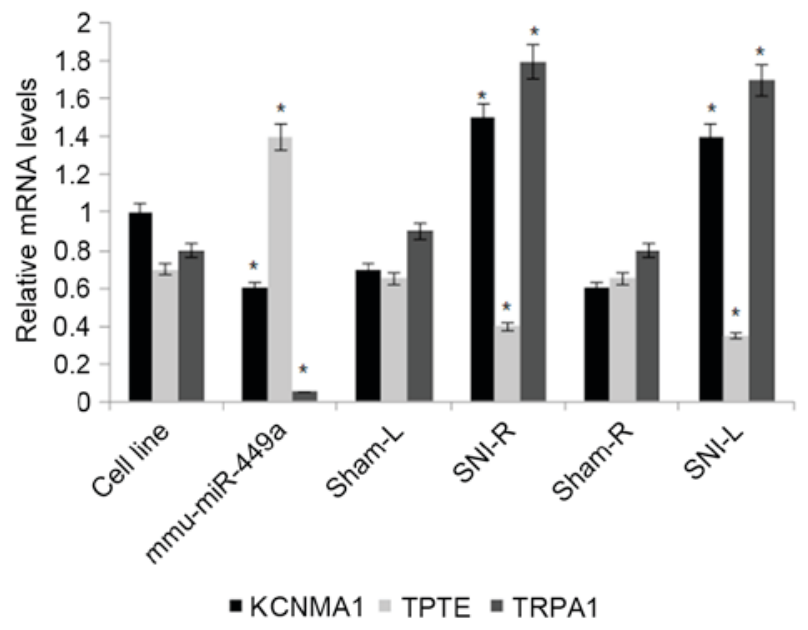

Figure 5. Reverse transcription-quantitative polymerase chain reaction analysis of the mRNA expression levels of TRPA1, KCNMA1 and TPTE in non-transfected and mmu-miR-449a-transfected cell line, SNI models and sham controls. $\beta$-actin served as an internal control. Data are presented are presented as the mean \pm standard deviation $(n=5) .{ }^{*} \mathrm{P}<0.05$ vs. the respective sham group. mmu, Mus musculus; miR, microRNA; TRPA1, transient receptor potential cation channel subfamily A member 1; KCNMA1, calcium-activated potassium channel subunit $\alpha-1$; TPTE, transmembrane phosphatase with tension homology; miR, microRNA; L, left; R, right.

P2X purinoceptor 5 (P2RX5), in DRG cells compared with non-transfected cells (Fig. 4). These results suggested that mmu-miR-185 does not serve an important role in regulating the levels of the majority of ion channel proteins.

Mmu-miR-449a affects the mRNA expression levels of TRPA1, KCNMA1 and TPTE. The effects of mmu-miRNA-449a on the ion channel protein levels of TRPA1, KCNMA1 and TPTE were assessed in DRG cells by RT-qPCR. Of the three proteins examined, TRPA1 demonstrated the most significantly altered mRNA expression levels between mmu-miRNA-449a-transfected cells and non-transfected cells, and between SNI and sham cells. TRPA1 mRNA expression levels significantly decreased in mmu-miRNA-449a-transfected cells and increased in the SNI models $(\mathrm{P}<0.05)$. KCNMA1 mRNA levels were additionally differentially expressed between mmu-miRNA-449a-transfected cells and non-transfected cells, and between SNI and sham cells. Its mRNA expression levels were additionally significantly increased in the SNI models $(\mathrm{P}<0.01$; Fig. 5). TPTE mRNA levels were differentially expressed between mmu-miRNA-449a-transfected and non-transfected cells $(\mathrm{P}<0.05)$, and were increased in mmu-miRNA-449a-transfected cells and decreased in SNI models $(\mathrm{P}<0.05)$.

\section{Discussion}

The results of the present study demonstrated that two microRNAs in the SNI models and sham mice were significantly downregulated: mmu-miR-449a and -185 . mmu-miR-185 overexpression did not affect the levels of most ion channel proteins, except P2RX5. P2RX5 is expressed in neurons, smooth and cardiac muscle cells, and leukocytes (35). In contrast, the overexpression of mmu-miR-449a affected the levels of most ion channel proteins. This result suggested that the peripheral deletion of mmu-miR-449a enhances novice inputs in DRG cells. Therefore, downregulation of mmu-miR-449a may be sufficient to affect the global pain mechanisms in SNI mouse models.

MiR-449a has been demonstrated to serve as a tumor suppressor in various cancers by regulating cell differentiation, particularly in neuroblastoma (36). miR-449a has additionally been revealed to be involved in the executive functioning of the brain, as demonstrated by the Wisconsin Card Sorting Test (37). However, the association between miR-449A and neuropathic pain remains to be elucidated. Therefore, the present study selected to use mmu-miR-449a. The present research provided evidence that mmu-miR-449a has a strong effect on neuronal excitability in DRG neurons, and that downregulation of the regulator contributes to pain hyposensitivity. This was primarily due to the upregulation of the channel protein TRAP1 in DRG cells.

Peripheral neuropathic pain in different disorders may be caused by various mechanisms and multiple etiological elements. In the present study, a unilateral SNI model was successfully established by a common traumatic nerve injury, which induces neuropathic pain. Although the model mimics the mechanisms of other types of neuropathic pain, its long-lasting behavioral mechanical hypersensitivity renders it useful for research on the underlying molecular mechanisms.

In the neuropathic pain model, mmu-miR-449a expression was observed to be significantly decreased in DRG neurons, with a dysregulation in levels of the ion channels proteins TRPA1, KCNMA1 and TPTE. Among these ion channel proteins, TRPA1 and KCNMA1 were markedly increased, whereas TPTE was significantly downregulated. Furthermore, these results demonstrated that increased expression of mmu-miR-449A caused the reverse changing trends of TRPA1, KCNMA1 and TPTE. Ionic channels serve important functions in sensory neurons, including transducing specific stimuli via electrical signals.

The results of the present study demonstrated that downregulation of mmu-miR-449a caused upregulation of TRPA1 protein expression levels in the SNI model, and may result in hypersensitivity to neuropathic pain. TRPA1 is an important member of transient receptor potential of cation channels family, and primarily exists in the sensory neurons of the trigeminal nerve and DRG cells (38). Furthermore, TRPA1 serves an important role in mechanical and norepinephrine hypersensitivity to neuropathic pain following nerve injury and may attenuate hyperalgesia $(39,40)$. Therefore, mmu-miR-449a may relieve neuropathic pain by down-regulating the levels of TRPA1.

KCNMA1 may be associated with neuropathic pain as increasing studies on potassium channel physiology have demonstrated the potential for the development of analgesics (33). Potassium channels have been reported to be associated with severe persistent breast pain following surgery (41). Similar to TRPA1, the results of the present study additionally revealed that KCNMA1 levels increased in SNI models and decreased in sham controls, and mmu-miR-449a effectively decreased the levels of KCNMA1.

There are certain limitations to the present study. Despite the effective establishment of the SNI mouse model, the precise molecular mechanisms underlying the downregulation 
of mmu-miR-449a and neuropathic pain remains unclear. mmu-miR-449a silencing or overexpressing animal models should be established to confirm the functional roles of mmu-miR449. In addition, two animals in each group are a small population and the results may be unstable for microarray analysis. To reduce the bias caused by the limited sample size, RT-qPCR analysis was repeated on eight further mice; the SNI and sham procedures were performed as aforementioned (data not shown), and this demonstrated similar results. Furthermore, assessing a broader range of ion channels may have revealed additional differential expression levels. The present work was limited to a mouse model; a rat model may be more representative due to its larger size.

In conclusion, in the present study, an SNI-mediated decrease in mmu-miR-449a levels suggested the functional importance of the microRNAs in trauma-induced neuropathic pain. Lower levels of mmu-miR-449a led to an increase in the ion channel proteins TRPA1 and KCNMA1, and a decrease in TPTE in DRG neurons, which was associated with the development of mechanical allodynia. In contrast, overexpression of mmu-miR-449a led to a decrease in expression levels of TRPA1 and KCNMA1, and an increase in TPTE in DRG neurons. From these results, mmu-miR-449a may be a potential therapeutic molecule for the alleviation of neuropathic pain.

\section{References}

1. Trost Z, Zielke M, Guck A, Nowlin L, Zakhidov D, France CR and Keefe F: The promise and challenge of virtual gaming technologies for chronic pain: The case of graded exposure for low back pain. Pain Manag 5: 197-206, 2015.

2. Gerges FJ, Manchanda C, Novak G, Al-Kimawi M, Semenovski M and Williams S: Occult spinal dysraphism: A challenge in pain management. Pain Physician 18: E225-E228, 2015.

3. Rogachov A, Cheng JC and DeSouza DD: Discriminating neural representations of physical and social pains: How multivariate statistics challenge the 'shared representation' theory of pain J Neurophysiol 114: 2558-2560, 2015.

4. Baquie P, Fooks L, Pope J and Tymms G: The challenge of managing mid-foot pain. Aust Fam Physician 44: 106-111, 2015.

5. Hayes K and Gordon DB: Delivering quality pain management: The challenge for nurses. AORN J 101: 328-334; quiz 335-337, 2015.

6. Foster DC, Falsetta ML, Woeller CF, Pollock SJ, Song K, Bonham A, Haidaris CG, Stodgell CJ, Messing SP, Iadarola M and Phipps RP: Site-specific mesenchymal control of inflammatory pain to yeast challenge in vulvodynia-afflicted and pain-free women. Pain 156: 386-396, 2015.

7. Frisch S: Perceptions of pain. Cultural differences add to the challenge of treating patients' pain. Minn Med 97: 14-16, 2014.

8. Dale O, Moksnes K and Kaasa S: European palliative care research collaborative pain guidelines: Opioid switching to improve analgesia or reduce side effects. A systematic review. Palliat Med 25: 494-503, 2011.

9. Rosenbaum D, Dallongeville J, Sabouret P and Bruckert E: Discontinuation of statin therapy due to muscular side effects: A survey in real life. Nutr Metab Cardiovasc Dis 23: 871-875, 2013.

10. Caires R, Luis E, Taberner FJ, Fernandez-Ballester G, Ferrer-Montiel A, Balazs EA, Gomis A, Belmonte C and de la Peña E: Hyaluronan modulates TRPV1 channel opening, reducing peripheral nociceptor activity and pain. Nat Commun 6: 8095,2015

11. Dib-Hajj SD, Black JA and Waxman SG: NaV1.9: A sodium channel linked to human pain. Nat Rev Neurosci 16: 511-519, 2015.

12. Cheng CF, Wang WC, Huang CY, Du PH, Yang JH and Tsaur ML: Coexpression of auxiliary subunits KChIP and DPPL in potassium channel Kv4-positive nociceptors and pain-modulating spinal interneurons. J Comp Neurol 524: 846-873, 2015.
13. Skerratt $\mathrm{SE}$ and West $\mathrm{CW}$ : Ion channel therapeutics for pain. Channels (Austin) 9: 344-351, 2015.

14. Sherkheli MA, Schreiner B, Haq R, Werner M and Hatt H: Borneol inhibits TRPA1, a proinflammatory and noxious pain-sensing cation channel. Pak J Pharm Sci 28: 1357-1363, 2015.

15. Laedermann CJ, Cachemaille M, Kirschmann G, Pertin M, Gosselin RD, Chang I, Albesa M, Towne C, Schneider BL, Kellenberger S, et al: Dysregulation of voltage-gated sodium channels by ubiquitin ligase NEDD4-2 in neuropathic pain. J Clin Invest 123: 3002-3013, 2013.

16. Sakai A and Suzuki H: microRNA and Pain. Adv Exp Med Biol 888: 17-39, 2015.

17. Linnstaedt SD, Walker MG, Parker JS, Yeh E, Sons RL, Zimny E, Lewandowski C, Hendry PL, Damiron K, Pearson C, et al: MicroRNA circulating in the early aftermath of motor vehicle collision predict persistent pain development and suggest a role for microRNA in sex-specific pain differences. Mol Pain 11: 66 , 2015.

18. Mallik S and Maulik U: MiRNA-TF-gene network analysis through ranking of biomolecules for multi-informative uterine leiomyoma dataset. J Biomed Inform 57: 308-319, 2015.

19. Galicia-Vázquez G, Chu J and Pelletier J: eIF4AII is dispensable for miRNA-mediated gene silencing. RNA 21: 1826-1833, 2015.

20. Ding M, Li J, Yu Y, Liu H, Yan Z, Wang J and Qian Q: Integrated analysis of miRNA, gene and pathway regulatory networks in hepatic cancer stem cells. J Transl Med 13: 259, 2015.

21. Yan L, Lee S, Lazzaro DR, Aranda J, Grant MB and Chaqour B: Single and Compound Knockouts of MicroRNA (miRNA)-155 and its Angiogenic Gene Target CCN1 in Mice Alter Vascular and Neovascular Growth in the Retina via Resident Microglia. J Biol Chem 290: 23264-23581, 2015.

22. van Spronsen M, van Battum EY, Kuijpers M, Vangoor VR, Rietman ML, Pothof J, Gumy LF, van Ijcken WF, Akhmanova A, Pasterkamp RJ and Hoogenraad CC: Developmental and activity-dependent miRNA expression profiling in primary hippocampal neuron cultures. PLoS One 8: e74907, 2013.

23. Zhang Y, Ueno Y, Liu XS, Buller B, Wang X, Chopp M and Zhang ZG: The microRNA-17-92 cluster enhances axonal outgrowth in embryonic cortical neurons. J Neurosci 33: 6885-6894, 2013

24. Impey S, Davare M, Lesiak A, Fortin D, Ando H, Varlamova O, Obrietan K, Soderling TR, Goodman RH and Wayman GA: An activity-induced microRNA controls dendritic spine formation by regulating Rac1-PAK signaling. Mol Cell Neurosci 43: 146-156, 2010.

25. Kusuda R, Cadetti F, Ravanelli MI, Sousa TA, Zanon S, De Lucca FL and Lucas G: Differential expression of microRNAs in mouse pain models. Mol Pain 7: 17, 2011.

26. Wang W, Shi Q, Mattes WB, Mendrick DL and Yang X: Translating extracellular microRNA into clinical biomarkers for drug-induced toxicity: From high-throughput profiling to validation. Biomark Med 9: 1177-1188, 2015.

27. Asha S, Sreekumar S and Soniya EV: Unravelling the complexity of microRNA-mediated gene regulation in black pepper (Piper nigrum L.) using high-throughput small RNA profiling. Plant Cell Rep 35: 53-63, 2016.

28. Richner M, Bjerrum OJ, Nykjaer A and Vaegter CB: The spared nerve injury (SNI) model of induced mechanical allodynia in mice. J Vis Exp pii: 3092, 2011.

29. Scroggs RS, Todorovic SM, Anderson EG and Fox AP: Variation in IH, IIR and ILEAK between acutely isolated adult rat dorsal root ganglion neurons of different size. J Neurophysiol 71: 271-279, 1994.

30. Zima V, Witschas K, Hynkova A, Zimová L, Barvík I and Vlachova V: Structural modeling and patch-clamp analysis of pain-related mutation TRPA1-N855S reveal inter-subunit salt bridges stabilizing the channel open state. Neuropharmacology 93: 294-307, 2015

31. Kanthesh BM, Sandle GI and Rajendran VM: Enhanced K(+) secretion in dextran sulfate-induced colitis reflects upregulation of large conductance apical $\mathrm{K}(+)$ channels (BK; Kcnma1). Am J Physiol Cell Physiol 305: C972-C980, 2013.

32. Tsantoulas C: Emerging potassium channel targets for the treatment of pain. Curr Opin Support Palliat Care 9: 147-154, 2015.

33. Pereira V, Busserolles J, Christin M, Devilliers M, Poupon L, Legha W, Alloui A, Aissouni Y, Bourinet E, Lesage F, et al: Role of the TREK2 potassium channel in cold and warm thermosensation and in pain perception. Pain 155: 2534-2544, 2014. 
34. Livak KJ and Schmittgen TD: Analysis of gene expression data using real-time quantitative PCR and the 2(-Delta Delta C(T)) method. Methods 25: 402-408, 2001.

35. Abramowski P, Ogrodowczyk C, Martin R and Pongs O: A truncation variant of the cation channel P2RX5 is upregulated during T cell activation. PLoS One 9: e104692, 2014.

36. Zhao Z, Ma X, Sung D, Li M, Kosti A, Lin G, Chen Y, Pertsemlidis A, Hsiao TH and Du L: microRNA-449a functions as a tumor suppressor in neuroblastoma through inducing cell differentiation and cell cycle arrest. RNA Biol 12: 538-554, 2015.

37. Lai CY, Yu SL, Hsieh MH, Chen CH, Chen HY, Wen CC, Huang YH, Hsiao PC, Hsiao CK, Liu CM, et al: MicroRNA expression aberration as potential peripheral blood biomarkers for schizophrenia. PLoS One 6: e21635, 2011.

38. Nassini R, Materazzi S, Benemei S and Geppetti P: The TRPA1 channel in inflammatory and neuropathic pain and migraine. Rev Physiol Biochem Pharmacol 167: 1-43, 2014.
39. Kogure W, Wang S, Tanaka K, Hao Y, Yamamoto S, Nishiyama N, Noguchi K and Dai Y: Elevated H2 O2 levels in trinitrobenzene sulfate-induced colitis rats contributes to visceral hyperalgesia through interaction with the ransient receptor potential ankyrin 1 cation channel. J Gastroenterol Hepatol 31: 1147-1453, 2016.

40. DeBerry JJ, Saloman JL, Dragoo BK, Albers KM and Davis BM: Artemin immunotherapy is effective in preventing and reversing cystitis-induced bladder hyperalgesia via TRPA1 regulation. J Pain 16: 628-636, 2015.

41. Langford DJ, Paul SM, West CM, Dunn LB, Levine JD, Kober KM, Dodd MJ, Miaskowski C and Aouizerat BE: Variations in potassium channel genes are associated with distinct trajectories of persistent breast pain after breast cancer surgery. Pain 156: 371-380, 2015. 